\title{
THE MICROSOFT GLOBAL ORTHO PROGRAM
}

\author{
Wolfgang Walcher ${ }^{\mathrm{a}}$, Franz Leberl ${ }^{\mathrm{b}}$, Michael Gruber $^{\mathrm{c}}$ \\ ${ }^{\text {a }}$ Microsoft Bing Imagery Technologies, $190015^{\text {th }}$ Street, Boulder, Colorado, wwalcher@ microsoft.com \\ ${ }^{\mathrm{b}}$ Graz University of Technology, Inst. of Computer Graphics and Vision, Austria \\ ${ }^{\mathrm{c}}$ Microsoft Bing Imagery Technologies, Graz, Austria
}

Commission IV, WG IV/2 - Automatic Geospatial Data Acquisition and Image-Based Databases

KEY WORDS: Transnational, Photogrammetry, Automation, Ortho-Rectification, Aerial Camera, Digital Elevation Model

\begin{abstract}
:
Wide area and thus continental mapping extending beyond national borders is a novel concept in civilian photogrammetry. The Microsoft Global Ortho Program was launched in the Spring of 2009 as a result of Microsoft's need for global geo-data at a high geometric resolution and radiometric excellence. By fall of 2012 more than 10 million $\mathrm{km}^{2}$ of the USA and 14 European countries will have been covered by seamless $30 \mathrm{~cm}$ GSD color-, $60 \mathrm{~cm}$ GSD false-color infrared ortho-mosaics and a 1 meter GSD digital surface model. The ortho-maps are being published to Microsoft's Bing Maps Internet mapping portal.
\end{abstract}

The Global Ortho Program was designed for highly and unprecedented automated mapping of essentially entire continents. In 2011, exclusive of flight operations, the product output per person has been measured in excess of 275,000 square km per year. We describe research efforts that made this achievement possible. Those include a specially designed aerial sensor (Ultracam G), logistics simulation for fight planning and optimization, in-flight blur detection and subsequent automatic blur removal, modeling and removal of atmospheric and environmental conditions, automated shear detection and DTM refinement, an IT architecture to process > 200,000 aerial images/day, and for creating over 1,000,000 $\mathrm{km}^{2}$ ortho-imagery and DSM data in 24 hours.

While addressing these issues, we provide ideas how this might affect the future of spatial infrastructure initiatives.

\section{Motivation for the Global Ortho Program}

Online mapping portals like Microsoft Bing Maps have distinguished themselves from earlier services by an imageryrich global experience. Users are expecting high-resolution, recent imagery of any place in the world. With commercial satellite imagery being restricted to resolutions of $50 \mathrm{~cm}$ or less, aerial imagery may be preferable. However, such coverage has not been very conducive to global applications. This, in 2009, triggered a Microsoft-decision for the Global Ortho Program. The goal: Cover the USA and Western Europe with $30 \mathrm{~cm}$ resolution orthophoto imagery in 2 years or less. The Bing Maps Imagery Technologies team (BITs) in Boulder, Colorado was tasked with executing the program.

Global Ortho is not a one-off project; it plans to extend into new territories and continuously update aging imagery.

\section{Program Scope}

Phase 1 of the Global Ortho Program covers the 48 states of the conterminous USA and 14 countries in Western Europe. Figure 1 (USA) and Figure 2 (EU) show the respective maps overlaid with the $1^{\circ} \times 1^{\circ}$ cell grid [see section 3]. Phase-1 comprises close to 1,400 complete and partial $1^{\circ}$-cells with $\sim 10.4$ mio km${ }^{2}$ of land. By April 2012 the program covered > 99\% of the US and $66 \%$ of the European terrain, well ahead of schedule.

Transnational aerial mapping of this scale has no precedence, although one can assume that there are past or present nonpublicized governmental satellite mapping projects of even larger size and much higher costs like the $\$ 7.3$ Billion EnhancedView contract awarded by US National GeospatialIntelligence Agency (NGA) [EnhancedView, 2012]

Aerial mapping programs could be related to one another via the number of pixels generated. In 2011, Global Ortho created $8,400,000 \mathrm{~km}^{2}$ of in-spec $30-\mathrm{cm}$ orthomosaics, Color InfraRed (CIR) mosaics and other by-products. The 2011 Global Ortho pixels were $>30$ times bigger than the average annual output of the USA National Agriculture Imagery Program (NAIP) [NAIP, 2009]. That program creates $1 \mathrm{~m}$ GSD color imagery of about one third of the continental USA every year. At one pixel per square meter, this equates to a multi-year average of about 3 trillion pixels per year. For comparison, in calendar year 2011, Global Ortho was creating 112 trillion pixels. This very likely makes Global Ortho the largest commercial aerial mapping program to date.

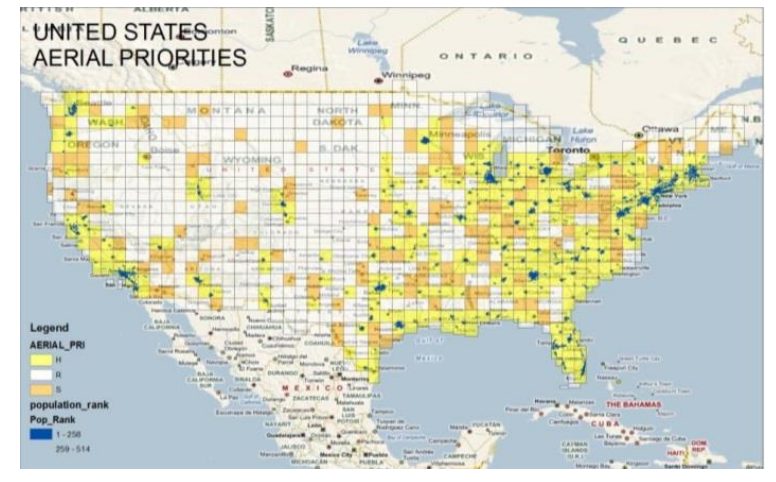

Figure 1: $1^{\circ}$ grid in USA. Colors describe priorities by population density (H-high, S-standard, R-rural).

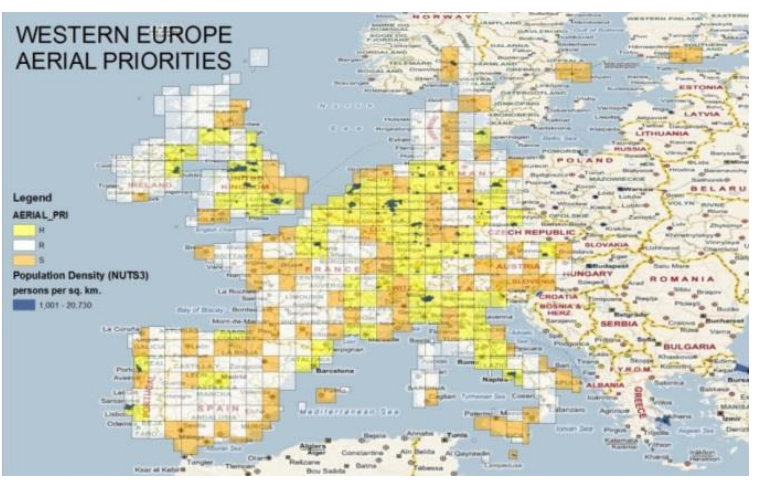

Figure 2: $1^{\circ}$ grid in EU. Colors as in Figure 1 


\section{Program Logistics and Aerial Acquisition}

Irregular national borders complicate any orthophoto project. Global/transnational coverage supports efficiency. Therefore the well- established $1^{\circ} \times 1^{\circ}$ grid as defined by the US-NGA serves well to spatially organize the Global program. The $1^{\circ}$ cell or "block" is a production unit with an average area of $\sim 8,000 \mathrm{~km}^{2}$. All specifications, planning, processing, quality control and product publishing are based on those blocks. Partial cells exist near water and along denied areas.

The North-South dimension of each cell is approximately 111 $\mathrm{km}$ while East-West dimensions decrease with latitude. Flight direction is standardized to $\mathrm{N}-\mathrm{S}$ unless terrain or airspace restrictions force a different pattern. Flight plans minimize the number of flight lines for a cell. High forward overlaps at $85 \%$ are needed for fully automated aerotriangulation and dense image matching. Therefore, a typical cell will be covered by 12 to $20 \mathrm{~N}$-S flight lines producing $\sim 1,700$ exposures.

Population density causes slight variations in specifications. About $10 \%$ of the areas are urban with denser flight lines to minimize building lean in ortho-products [thus High, Standard and Rural population densities in Figs 1 and 2].

To collect imagery per cell within a single 6-hour-aerial sortie, the novel UltraCam-G [UC-G] camera was built [section 5]. High productivity also was made possible by means of a novel project simulation tool. Individual airplane characteristics, flight plan (altitude, lines, turns, etc.), historic weather patterns, daily sun angle, leaf on-off seasons, seasonal snow cover, technical, logistical and localized environmental factors enter into scenarios for the forecast of a globally optimized solution.

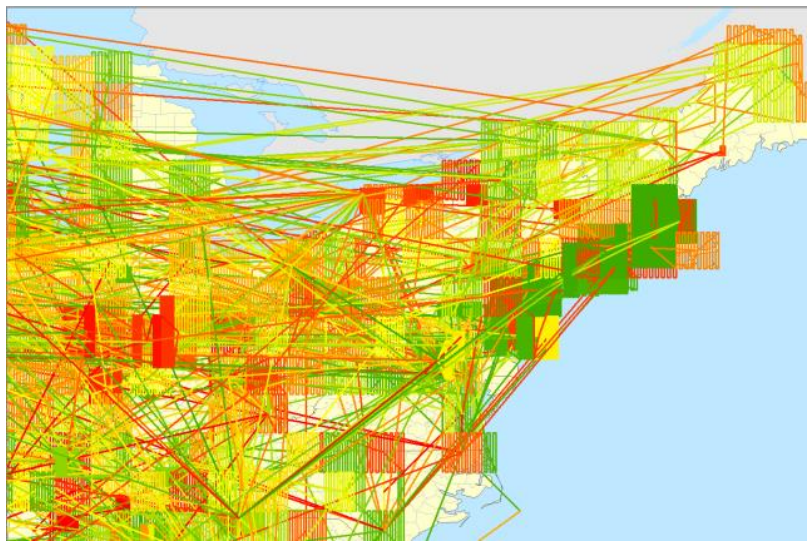

Figure 3: Simulated flight pattern over the N-E US for a 6-month period. Color indicates different flight dates.

The forecast over a period of 12 months has proven to be within $\pm 4 \%$ of actual acquisition totals. As a result of this optimization, only 12 UC-Gs, operated by experienced regional aerial photography firms, were required for all aerial work. These UC-Gs and aircraft collected > $1 \mathrm{mio} \mathrm{km}^{2}$ in Oct 2011.

\section{Global Ortho Data Products}

Global Ortho produces a $30 \mathrm{~cm}$ RGB true color and $60 \mathrm{~cm}$ false color infrared (CIR) orthophoto (Figs 4, 5). A digital surface model DSM [and DTM] gets created for use in image rectification. Only the RGB-product is published to Bing Maps.

The $1^{\circ}$ grid, as the smallest unit of data production, is a seamless image mosaic with a 200 meter buffer into adjacent cells or bodies of water. Each color-ortho has a twin CIR mosaic, even though at a lower resolution $[1$ CIR-pixel $=4$ RGB-pixels]. The CIR product is not published to Bing Maps but can be purchased from DigitalGlobe.

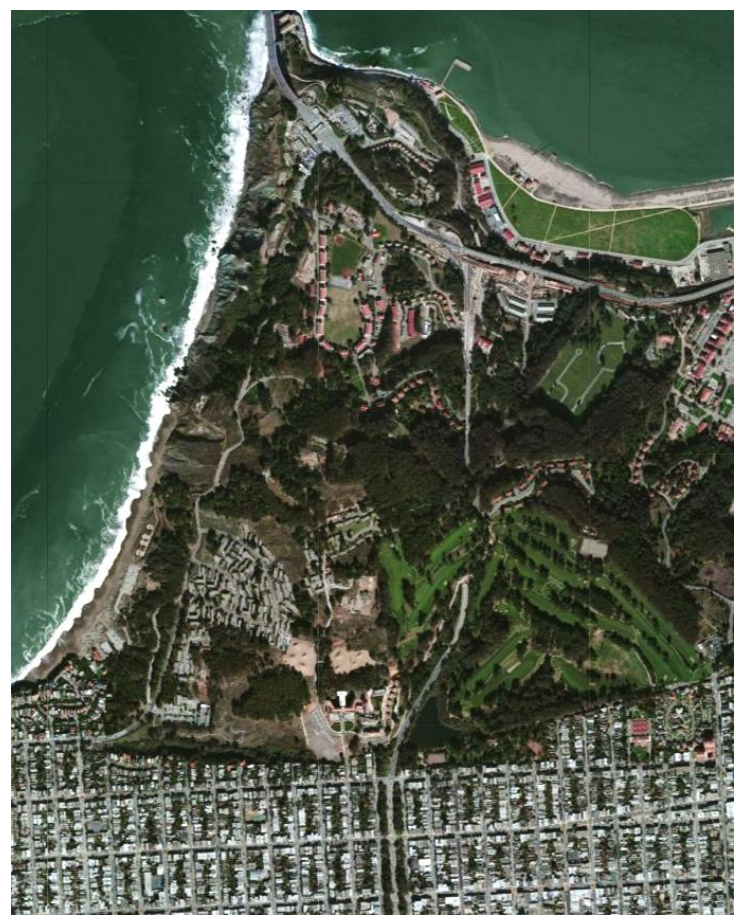

Figure 4: Color RGB orthophoto mosaic, Presidio in San Francisco, CA. Area shown is $~ 2.5 \mathrm{~km}$ by $3.0 \mathrm{~km}$

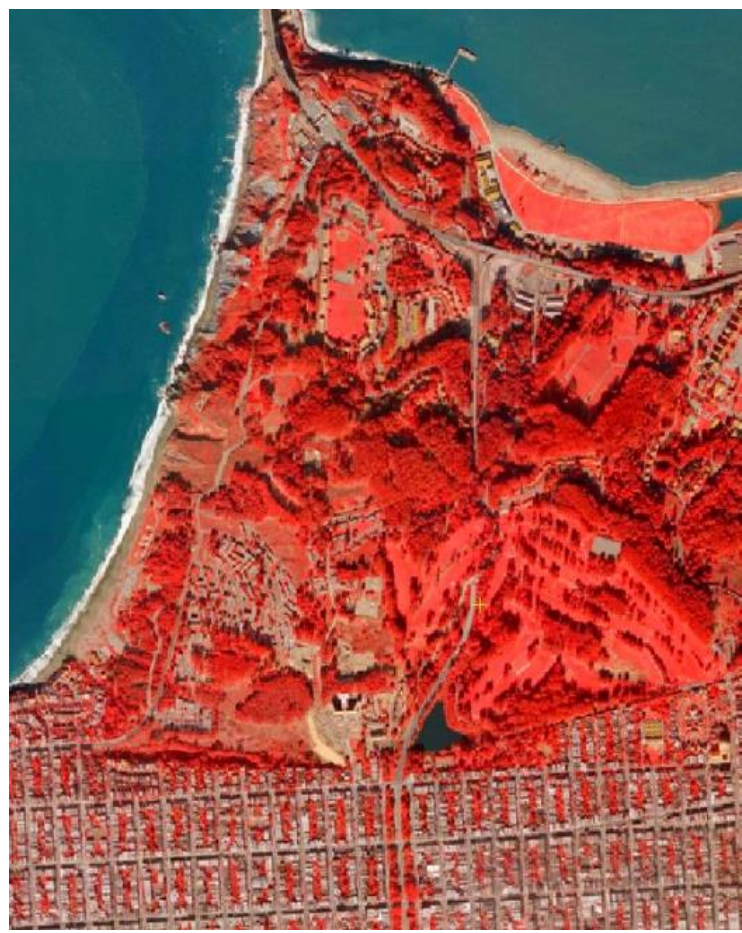

Figure 5: Color Infrared (CIR) ortho mosaic of area in Fig.4

A main achievement has been the level of automation of production processes while meeting specifications in radiometry, geometry and environment. This needed automated Quality Control (QC) and equally automated problem fixes. This in turn required all specifications to be quantifiable and 
measureable in a way that can be implemented in computer programs. At the same time the old adage applies, namely that $100 \%$ perfection is impossible to achieve. Conversion of standard aerial trade practices into computer code reveals their often very vague language, and that expectation of flawless perfection is unrealistic.

A discussion of all quality measures and specifications is beyond this publication. However, we can describe the most important specification, namely on absolute positional accuracy. Table 1 below lists the minimum requirements for absolute horizontal errors. It is important to mention that to date, with $90 \%$ program completion, all tests so far have shown an RMS error of less than \pm 2 meter in $\mathrm{x}$ and $\mathrm{y}$ directions.

\begin{tabular}{|c|c|c|}
\hline $\begin{array}{c}\text { Block } \\
\text { Designation }\end{array}$ & $\begin{array}{c}\text { CE95 Accuracy } \\
\text { Requirements }\end{array}$ & $\begin{array}{c}\sim \text { Equivalent } \\
\text { RMSE in X and Y }\end{array}$ \\
\hline High Population & $10 \mathrm{ft} / 3 \mathrm{~m}$ & $4 \mathrm{ft} / 1.2 \mathrm{~m}$ \\
\hline Standard & $15 \mathrm{ft} / 4.5 \mathrm{~m}$ & $6 \mathrm{ft} / 1.8 \mathrm{~m}$ \\
\hline Remote & $20 \mathrm{ft} / 6 \mathrm{~m}$ & $8 \mathrm{ft} / 2.4 \mathrm{~m}$ \\
\hline
\end{tabular}

Table 1: Minimum absolute positional accuracy requirements by class of block. In practice, to date all "Remote" blocks have meet or exceeded the "Standard" accuracy requirement.

The vertical errors of the DSM affect the XY-accuracy, especially with a wide-angle UC-G. DSM postings are at $1 \mathrm{~m}$ (Figure 6). This in turn produces the DTM for orthorectification. The DSM is available from Bing Maps, the DTM data has not been released as a product.

The DSM is created from highly overlapping $75 \mathrm{~cm}$ resolution UC-G panchromatic images, using global optimization as it is currently being used globally [Hirschmüller, 2005, 2008]. The product is sampled at a $1 \mathrm{~m}$ grid spacing, but the true horizontal resolution is closer to $1.5 \mathrm{~m}$ in most cases. The vertical accuracy of the DSM has been measured at $\pm 1 \mathrm{~m}$ on solid surfaces using ground truth.

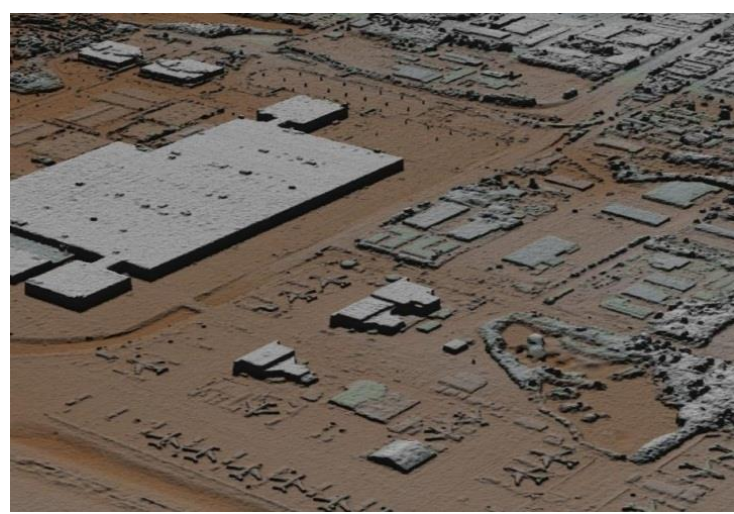

Figure 6: Visualization of $\sim 3 \mathrm{~km}^{2}$ near the Boeing facilities north of Seattle, WA as a detail from the nation-wide DSM. Point density at 1 $\mathrm{m}$; nominal vertical error is at $\pm 1 \mathrm{~m}$ on solid surfaces.

It is worth mentioning that the RGB, CIR, and DSM data are all created from the same UC-G images and are therefore perfectly aligned in time and space.

\section{UltraCam G: Aerial Camera for Large Area Orthos}

An aggressive program timeline and cost plan resulted in the development of a novel aerial camera concept in the form of the UC-G. While the stitching of large images from smaller tiles was retained, the novel camera inverts the role of panchromatic and color imaging and pan-sharpening [Weidner
Centeno, 2009]. A wide swath with almost 30K cross-track pixels and high photogrammetric elevation accuracy was achieved. The later was especially important to avoid the dependency on pre-existing elevation models for the creation of high-quality ortho products.

The UC-G came about in 2009 at Microsoft Photogrammetry in Graz, Austria. This team has a history of developing innovative aerial cameras [Leberl et al., 2003]. Figure 7 and 8 illustrate a 7cone camera with a larger format, but lower resolution panchromatic as well as a smaller format higher resolution color component. The panchromatic image is formed using the proven technology of the UltraCam Lp with 2 camera heads, using precision calibration [Ladstädter et al., 2010]. The focal length is at $40 \mathrm{~mm}$ for a relatively wide field of view of $80^{\circ}$ across the swath. This produces a strong photogrammetric solution and serves as the geometry backbone of the UC-G.

The color and IR components of the UC-G get collected via another 5 optical cones [as illustrated in Fig. 8] of which 4 are used for the RGB-data, one for the NIR-image.
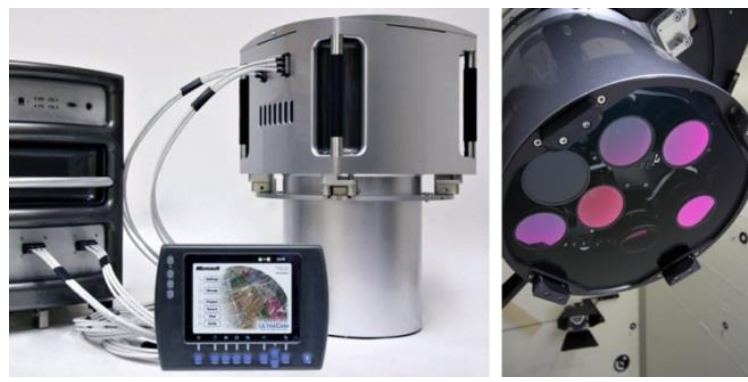

Figure 7: The UltraCam-G technology is based on the design of the UltraCam family (Senor Unit, Data Storage Unit and User Interface Panel) but has a new 7-cone concept shown on the right.

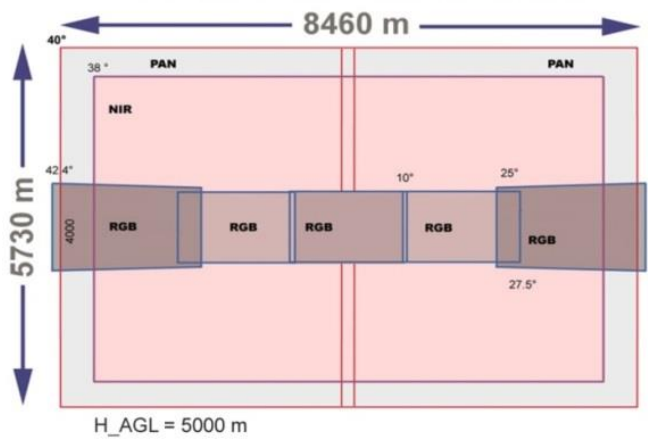

Figure 8: Frame format of the UltraCam-G. At a flying height above ground of $5000 \mathrm{~m}(16400 \mathrm{ft}$.) the swath covers $>8 \mathrm{~km}$. Pan and RGB cover the full swath, NIR has a slightly reduced format.

The panchromatic image has an aspect ratio 3:2. Two $40 \mathrm{~mm}$ optical lenses collect light onto a 90 Mpixel panchromatic area CCD array each.

The RGB color is a narrow rectangular frame at 28,200 pixels cross track and about 3,900 pixels along track. The RGB is registered to the panchromatic channel and covers the entire ground but at a smaller forward overlap. Figure 9 illustrates the 3 components (pan, RGB, NIR) of an UC-G image.

Global Ortho is flown from a height of $5000 \mathrm{~m}$ to achieve a Ground Sampling Distance (GSD) at $30 \mathrm{~cm}$ for RGB, $75 \mathrm{~cm}$ for pan and $125 \mathrm{~cm}$ for NIR images. The standard forward 
overlap of $85 \%$ for the pan-images results in a $10 \%$ forward overlap of the RGB images.
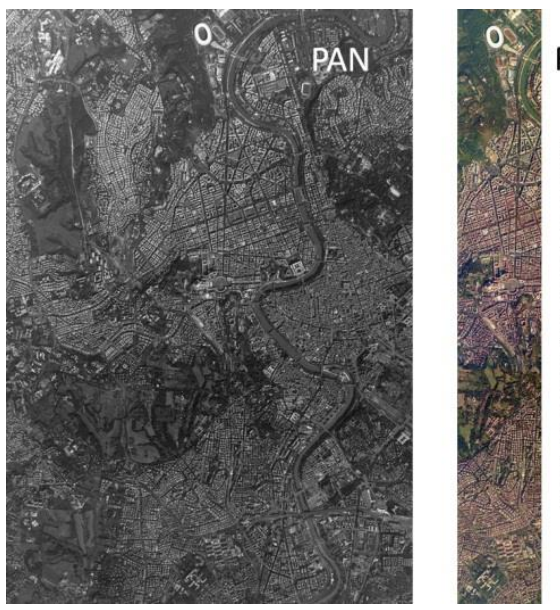

RGB

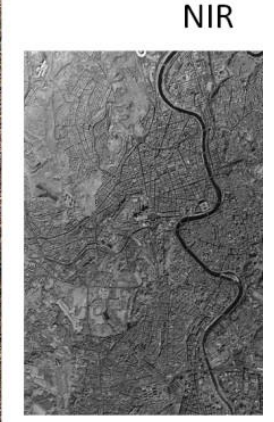

Figure 9: The 3 image planes of one UC-G frame showing the city of Rome, Italy -- flight direction from left to right. (NIR image is reduced)

\section{Ultracam-G Accuracy Experiment}

The horizontal system accuracy should be of the order of one color pixel $(30 \mathrm{~cm})$. To assess the UC-G capabilities, a test was performed in 2009 over Rome, Italy (Fig.10). The BINGO/UltraMap-workflow for aero-triangulation was applied to the high overlap panchromatic images with their $75 \mathrm{~cm}$ pixels. A $\sigma_{0}$ was achieved at 0.96 and the RMSE values of ground control points and check points was at \pm 0.4 GSD of the panchromatic camera, equal to $30 \mathrm{~cm}$. This geometric performance meets the hoped-for \pm 1 pixel of the higher resolution RGB image.

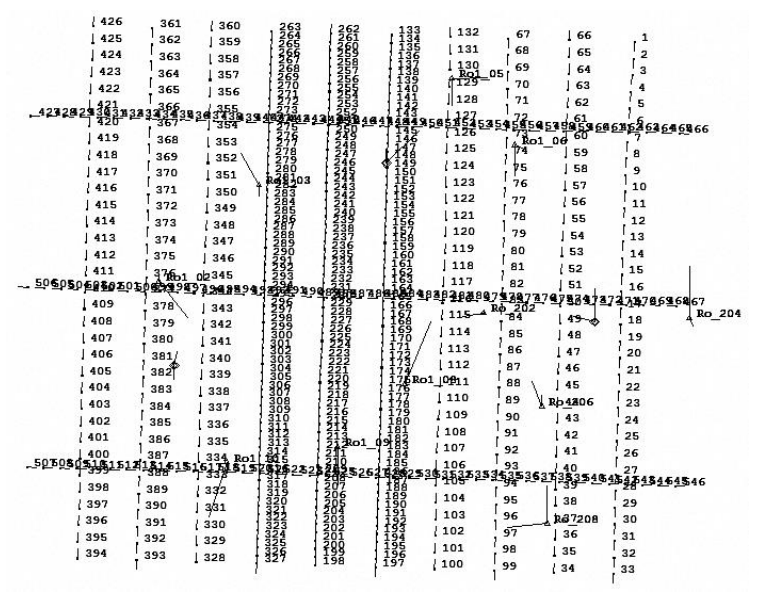

Figure 10: Flight layout in Rome [Italy], 546 images, 13 flight lines.

We conclude from those triangulation results that the DSM will support the horizontal accuracy for the RGB product specified in Table 1. Tests with ground truth confirm a vertical DSMerror at $\sim 1$ and 1.5 times the input pixel size: an RSM-error at $\pm 1 \mathrm{~m}$ gets achieved from $75 \mathrm{~cm}$ input pixels. The horizontal error due to relief and vertical objects in the RGB-orthos is largest at the edge of the field-of-view at $+/-40^{\circ}$ and roughly equal to the vertical error.

\section{About Manual Work versus Full Automation}

A core innovation is the level of automation that had a team of about 25 people create over 9 million $\mathrm{km}^{2}$ of ortho-imagery in a two years time frame. Automation requires large engineering investments, costly sensors and IT-infrastructure, but results not only in reduced variable costs, but also in predictability, repeatability and scalability of processes, and in the elimination or minimization of human error. The latter is especially significant with highly repetitive tasks. Figure 11 compares manually measured shears [geometric discrepancies along edges/seams of ortho-patches] with automatic measurements and shows a rather significant difference between manual and automated results.

Predictability: Given a set of input data one can foretell the expected properties of the output data along with timelines and resource consumption. This contrasts with approaches relying on "best business practices" or "commercially reasonable efforts".

Repeatability: Given a set of input data, multiple runs of the same process will create exactly the same results. This permits one to re-think entire workflows, for example with respect to intermediate data that can be recomputed instead of stored.

Scalability: Throughput should increase linearly with increased computing, storage and networking capacity.

In this context, automation as not a simple "translation to software" of established manual processes. Processes optimized for humans make the best possible use of human strengths and as a result they often require frequent and intermittent human judgment. By definition those judgment calls are complicated or even impossible to automate.

Instead, once a process is predictable, repeatable, and scalable, it is possible to automatically create multiple different versions of a data product simultaneously. Instead of making multiple human decisions during product creation, the human operator now simply looks at the resulting product variants, picks the most suitable one, and discards all others.

Automatic vs. Measured Shear at $12,000+$ Locations

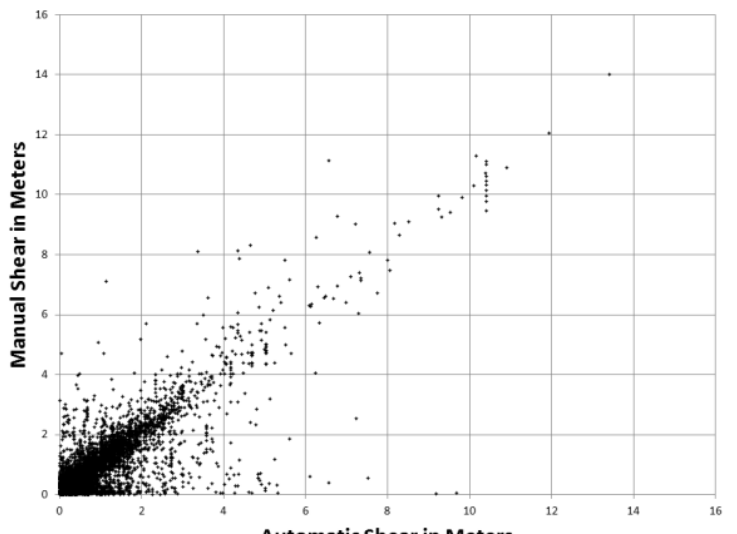

Automatic Shear in Meters

Figure 11: Comparison of over 12,000 manual measurements for image shear to the automated measurements. Offsets from the $\mathrm{x}=\mathrm{y}$ diagonal indicate human measurement errors.

A compute-heavy full automation has been applied throughout the Global Ortho Program. For example, it is used to determine optimal processing parameters for unusual environmental conditions like heavy smog with its effects on radiometry. An 
operator can use this process to determine optimal settings for such conditions and then train the system to use those settings for all subsequent grid cells with similar conditions. This approach is the very reason for the scale of the IT processing architecture described in the following section.

\section{Workflow Management and IT Environment}

Processing millions of aerial images for thousands of photogrammetric blocks, potentially multiple times each, does not only require an IT infrastructure of the highest levels. Even more important are tools for process scheduling and data management. For this, Microsoft has developed the proprietary Imagery Processing Framework (IPF). IPF is a general-purpose architecture and used by BITs for all Bing Maps processing and publishing. Principally, it reflects the technologies used by all global internet search providers for their various processing centers. In its core IPF has multiple very large distributed MS SQL Server databases to keep track of processes and data. Currently IPF manages over 2,500,000,000 individual files and 30 million current or past processing tasks along with metadata, hardware status and other vital system information.

IPF is implemented within one of Microsoft's data centers and accessed remotely from the BITs location in Boulder, Colorado, USA. The current hardware implementation is comprised of approx. 2,400 servers with 24,000 processor cores and 71 petabytes $(71,000,000$ gigabytes) of available disk storage space. Global Ortho does only require a relatively small percentage of the available processing and storage infrastructure. However, a peak processing capacity in excess of 1 million $\mathrm{km}^{2}$ of Global Ortho data per 24 hours has been achieved and is key to our approach to automation.

\section{Some Key Innovations}

The Global Ortho processing workflow follows the traditional sequence of aerial acquisition, aerotriangulation and bundle adjustment, DSM and DTM creation, orthorectification and mosaicking, radiometric processing, and final product formatting and QC. However, because of the requirements for scale and automation discussed earlier, the implementation of these individual steps in most cases required innovative approaches. A detailed discussion of all those innovations would far exceed the scope of this publication. Several BITs team members and Microsoft researchers are preparing a series of publications describing individual topics in some detail. Intellectual properties protection limits such publishing activity to patent-pending innovations. Below we highlight a few of those.

Motion Blur: To reduce the need of re-flying an area because of blurred imagery the UC-G software has a novel and unique inflight capability to detect camera motion that causes motion blur. The camera captures and stores in real-time all the parameters necessary to later algorithmically remove such motion blur from the imagery. The detailed description of this blur detection and removal feature can be found in [Schickler, Shah 2012].

Radiometric Adjustment: All image processing employs 16-bits per color band to make maximum use of the UltraCam's dynamic range of almost 14 bits. Conversion from the 16 to an 8 -bit color space is one of the very last processing steps. To create radiometrically uniform and well balanced output images, first all impactful atmospheric and lighting conditions are removed. Then tonal adjustment and color balancing is applied to all images of a block. Novel approaches for both processes have been developed by BITs in cooperation with Microsoft Research. Figures $12 \mathrm{a}$ and $12 \mathrm{~b}$ show the same $1^{\circ}{ }_{-}$ cell containing 1700 UC-G images before and after applying these processes. In this example, weather changes had resulted in several partial flight missions many days apart resulting in extreme radiometric differences (Figure 12a). Figure 12b shows that same cell after automated color balancing.

Specular Reflection: Similar algorithms have been developed to physically model, detect and then suppress the impact of specular reflection, especially on water. In traditional mapping processes such artifacts are often edited and removed by a labor intensive process. In the Global Ortho production process such reflections are almost entirely removed without any user interaction. Figure 13b shows the result of this automated process applied to the image shown in Figure 13a Figure 13a.

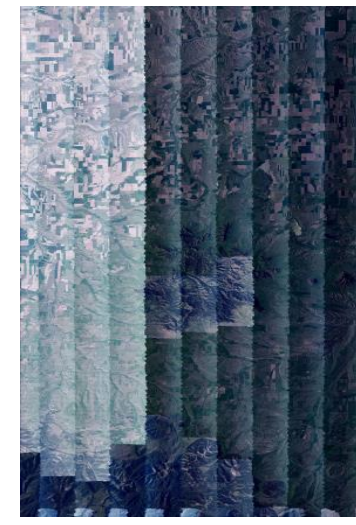

Figure 12a: $1^{\circ}$-cell $(\sim 8,500 \mathrm{~km} 2)$ before color balancing

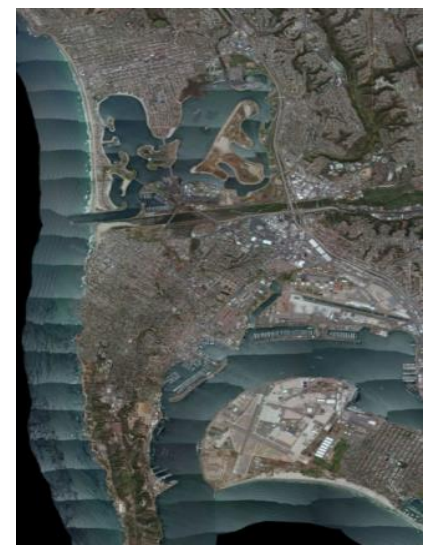

Figure 13a: Specular reflection on water near San Diego, CA, affecting each individual photo and flight line

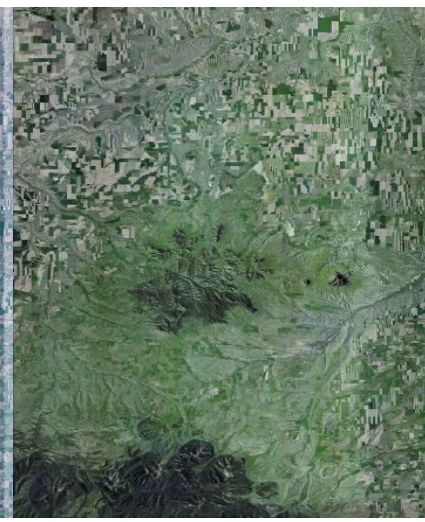

Figure 12b: The cell of Fig. 12a after automated color balancing

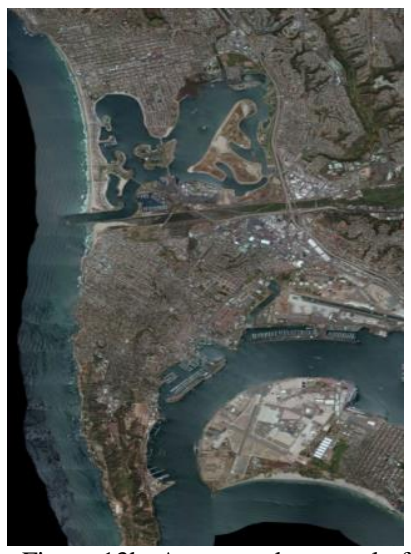

Figure 13b: Automated removal of specular reflections from water in each photo and flight line
Seamlessness: Many approaches exist to automatically place seam lines between ortho patches. However, the effort to visually inspect more than 11 million kilometers of seam lines to detect, and correct errors by potential mismatches between ortho patches (shear) would be astronomical. We therefore developed an automated method to measure the shear in all places where a seam line crosses a linear feature and we do this at sub-pixel accuracy. This allows automatic detection of product defects and spec violations, which can be used to trigger a number of automatic attempts to fix the problem, and in rare cases, call for human intervention. Figure 14 shows the distribution of all acceptable (green) and questionable (red) occurrences of shear over the larger Columbia, South Carolina metro area. 


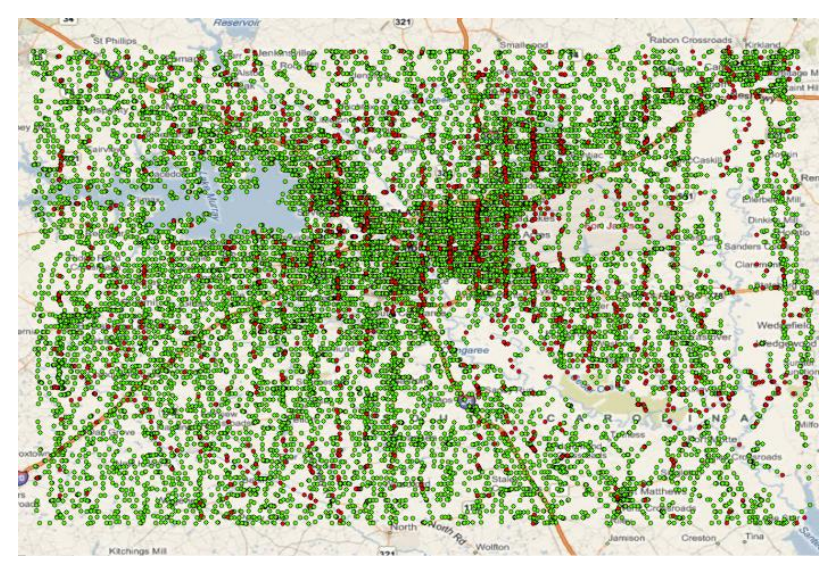

Figure 14: Automated shear analysis along seam lines. 2134 locations (red) out of over 30,000 (green) have questionable shear.

\section{Results}

A most significant achievement of the Global Ortho Program has been that today it is slightly ahead of schedule while far exceeding the initially expected quality specifications.

The program required a number of research questions to be resolved. These included the issue of efficiency and cost in sensing with a novel camera that promises color imagery of large areas with minimum relief displacement of buildings and vegetation, automation of aerial triangulation, dense DEMcreation and seamless orthophoto products from separately collected patches. It also required the setup of a large computing facility to process 200,000 input images for a project area of $1,000,000 \mathrm{~km}^{2}$ per 24-hour period.

The absolute horizontal accuracy of the RGB orthophotoproduct was expected to be within $\pm 1.2 \mathrm{~m} \mathrm{r.m.s}$. for urban, and $\pm 3 \mathrm{~m}$ for rural areas [representing \pm 3 to \pm 6 meter Circular Error at $95 \%$ probability]. Measurements have shown that the actual absolute horizontal accuracy r.m.s. is the range of $\pm 1 \mathrm{~m}$ not exceeding $\pm 2 \mathrm{~m}$. This horizontal accuracy can be achieved without the use of any survey ground control but with complete reliance on on-bard differential GPS and IMU- measurements. However, we use up to 4 control points per $1^{\circ}$-cell for reasons of quality assurance and to avoid vertical bias which could affect vertical accuracy of the DSM. Vertical accuracy of the DSM is found to be within $\pm 1 \mathrm{~m}$.

Throughput and productivity have not been limited by compute power or by human labor, but by the rate at which aerial imagery can be acquired. The IPF processing environment is capable of processing the entire imagery harvest of a whole year worth of flying in about one week. Given the current levels of quality control and inspection the annual production rate per person is between 300,000 and $400,000 \mathrm{~km}^{2}$. With only minor changes to the product specifications this rate could be increased even further. For example, applying "Standard" quality specifications to high population areas [see Table 1] would almost entirely eliminate the need for manual QC.

The Global Ortho Program's scale and aggressive timeline have triggered a number of innovations in hardware, software, logistic, processing and more. Many of these innovations are now being introduced in other BITs projects and will find their way into new generations of UltraCam sensors, UltraMap software [Reitinger et al., 2012], and Microsoft photogrammetry tools.

\section{Outlook}

Completion of the initial coverage for the US and Western Europe was originally expected for the early fall of 2012, thus within 30 months from kick off in March 2010. However, already starting in the summer of 2012, we also expect to begin a new project addressing updates of select areas on an asneeded basis. The main focus of Bing Maps is to extend the coverage footprint of the Global Ortho Program. This will, at least in part, be done in close cooperation with external partners. At the time of this writing several national and international organizations have engaged in talks about expanding Global Ortho into their areas of interest.

While this project explores potentials with most advanced computing architectures, algorithms, sensors and procedures, we all know that what is "most advanced" today, will be widely available tomorrow. Therefore such a project is a preview of what is to come to everyday photogrammetric workflows everywhere.

\section{References}

EnhancedView (2010) (http://www.spacedaily.com/reports/ GeoEye_Wins_National_Geospatial_Intelligence_Agency_Enh anced_View_Award_999.html. Last visited 19-Apr-2012.

Hirschmueller, H. (2005): Accurate and efficient stereo processing by semi-global matching and mutual information. Proceedings of the IEEE Conference on Computer Vision and Pattern Recognition, pp. 807-814

Hirschmueller, H. (2008): Stereo vision in structured environments by consistent semi-global matching. IEEE Transaction on Pattern Analysis and Machine Intelligence PAMI 30 (2) pp. 328-341.

Leberl, F. M. Gruber, M. Ponticelli, S. Bernoegger, and R. Perko (2003): The UltraCam Large Format Aerial Digital Camera System, Proceedings of the American Society for Photogrammetry \& Remote Sensing, 5-9 May, 2003, Anchorage, AL, unpaginated CD

Ladstaedter R., M. Gruber, A. Wiechert (2010): Monolithic Stitching: One Sensor Geometry For Multiple Sensor Cameras, Proceedings of the American Society for Photogrammetry \& Remote Sensing, 26-30 April, 2010, San Diego, CA, unpaginated DVD

NAIP (2009): http://www.fsa.usda.gov/Internet/FSA_File/
naip 2009 info final.pdf

Schickler W., C. Shah (2012): Automated Blur Detection and Removal in Airborne Imaging Systems using IMU Data. International Archives of the Photogrammetry, Remote Sensing and Spatial Information Sciences, Melbourne, Australia.

Reitinger B. (2012) UltraMap V3 - A Revolution in Aerial Photogrammetry. International Archives of the Photogrammetry, Remote Sensing and Spatial Information Sciences, Melbourne, Australia.

Weidner, U., J. Centeno (2009): Pansharpening - Simple Approaches and Their Evaluation. In: Photogrammetrie Fernerkundung - Geoinformation (PFG), 4/2009. 